\title{
SCALABLE AR FOR BIM ON TELECOMMUNICATION NETWORK SITES
}

\author{
V. Palma ${ }^{1}$, R. Spallone ${ }^{1}$, G. Cicone ${ }^{2}$, G. Lops ${ }^{2}$, R. Rinauro ${ }^{2}$ \\ ${ }^{1}$ Politecnico di Torino, Department of Architecture and Design, Italy - (valerio.palma, roberta.spallone)@polito.it \\ ${ }^{2}$ INWIT - Infrastrutture Wireless Italiane S.p.A., Italy - (giulia.cicone, gianpiero.lops, roberto.rinauro)@inwit.it
}

\section{Commission II}

KEY WORDS: AR, BIM, project management, telecommunications, markerless tracking

\begin{abstract}
:
A growing number of research works, experiments and applications is investigating the potential at the intersection of augmented reality (AR) and the architecture, engineering, and construction (AEC) industry. Project management, project communication, collaborative design, maintenance and construction progress documentation, construction site safety, and training are some of the cases that can benefit from blending real and virtual views through mobile devices. In recent years, research also highlighted how mixed reality and building information modeling (BIM) could cooperate to provide effective communication between multiple agents and closer interaction between digital information and the building site. Nevertheless, consolidated applications in these fields are still limited, especially when compared to other areas of AR adoption. This paper presents the development of an AR-based mobile app for exploring telecommunications tower sites and interacting with a related BIM database. The project aims to provide easy-to-use tools to maintain both the physical assets and an up-to-date model. We discuss critical issues in developing a scalable and interoperable application, supporting the feasibility study of similar solutions in the AEC sector.
\end{abstract}

\section{INTRODUCTION}

The architecture, engineering, and construction (AEC) sector increasingly consider augmented reality (AR) a design support tool (Garbett et al., 2021) and a growing number of experiments is investigating the potential at this intersection (Noghabaei et al., 2020). Nevertheless, consolidated applications are still limited, especially when compared to other areas of AR adoption.

AR tools ensure more intuitive exploration and understanding of 3D models. This increased usability of digital representation can boost applications in managing complex design projects and support maintenance, documentation, and professional training. By fostering bidirectional and frequent communication between the artifact and its representation, AR can contribute to the study and specialization of the digital twin paradigm - a wellestablished concept in the production engineering sector (Grieves and Vickers, 2017) - for architectural contexts (Khajavi et al., 2019).

The proposed contribution aims to clarify some operational possibilities related recent AR solutions, in order to develop more feasible and scalable applications. We present an ongoing project dealing with the integration of AR - or, more generally, of computer vision technologies to interact with the built space with building information modeling (BIM). The research team studied how this combination could facilitate the comparison between digital design and the construction site, and aid database updating operations. The application field is the management of an extensive network of sites for mobile communications towers and is based on the development of an app for mobile devices.

\section{RELATED WORKS}

Augmented Reality overlays digital layers to real-word images captured by a camera (Amin and Govilkar, 2015). The system generates a coherent perspective view of the virtual components by estimating the user's position and movements, thus providing intuitive understanding and interaction methods to access spacerelated information. AR is part of a broader domain of mixed reality (MR), which includes the many shades of the intersection between digital imagery and real-world stimuli (Milgram and Kishino 1994).

In recent years, the evolution of computer graphics and the commercial success of AR technologies have been spreading devices specifically designed to blend real space and digital objects. Smartphones and other hand-held devices integrate hardware that aids in mapping the user's location and surroundings, such as multiple cameras with different sized fields of view, motion sensors, AI chips for computer-vision tasks, and even LiDAR scanners (Campi at al., 2021). Furthermore, research on dedicated hardware in head-worn format, although still far from widespread use, suggests an increasing pervasiveness of AR in everyday life and professional activities (Lin et al., 2021).

In the AEC sector, AR shows advantages in addressing a variety of management issues (Ahmed, 2019). Project communication is often seen as a task that could benefit from immersive visualization. AR can induce a more intuitive comprehension of the project, thus supporting option evaluation, collaborative design, and the reception of end-user's preferences (Zaher et al., 2018; Noghabaei et al., 2020). Much research highlights the possible applications of mixed reality in construction safety, spanning hazard identification, safety inspection, instruction, and training (Li et al., 2018).

Support in the construction phases includes progress tracking, clash detection, and the on-site validation of the design. Several experimental projects discuss the advantages of AR in carrying out on-site, highly customized manufacturing or parametric design-related operations (Akbari, 2018; Jahn et al., 2019). As Song et al. (2021) argue, digital fabrication in architecture can exploit AR in guiding assemblage and construction procedures, enabling on-site customization and optimization, and handling design defects and construction tolerance. IT company Fologram demonstrated AR usage for 3D "holographic" instruction in a construction site environment, that is, showing 1:1 CAD drawings through headset devices (Felix, 2019). Conversely, a partnership between Autodesk and RealWear proposed AR access to BIM data - again exploiting hands-free devices without showing full-scale site-matching elements (RealWear, 
Inc., 2021). The actual integration of $3 \mathrm{D}$ models and nongeometric data seems still limited and is not supported out-ofthe-box by dedicated software.

In recent years, research on the interaction between MR and BIM has begun to highlight the complementarity of the two technological areas (Ahmed, 2019; Noghabaei et al., 2020). AR can in fact support the mitigation of some shortcomings related to the complexity of BIM models, such as communication between multiple agents with different skills and the interaction between digital information and the building site.

Williams et al. (2015) proposed one of the first methods for accessing BIM data - both geometric and informative - through mobile AR, addressing a single-model case and relying on a predefined observation spot for the alignment of physical space and the digital model. More recently, Garbett et al. (2021) proposed an AR system connected to a cloud database, again through marker-based solutions. A "markerless" experiment for the AEC area was proposed by Kopsida and Brilakis (2016), who superimposed a single BIM project to a camera scene, tracked using a Kinect sensor. Nevertheless, we have not identified markerless AR applications in scalable BIM-enabled projects.

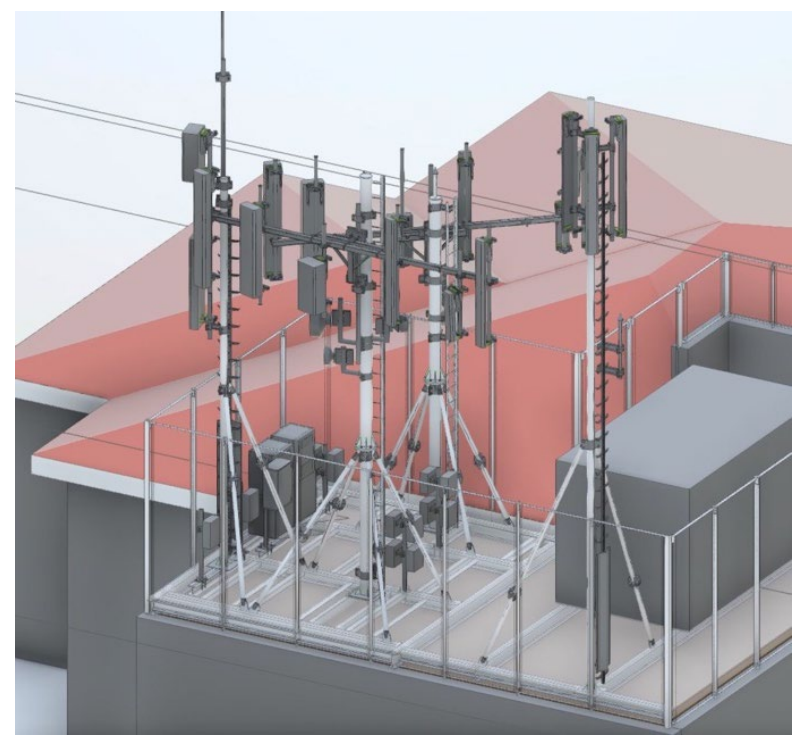

Figure 1. Axonometric view of the BIM representation of one of the sites managed by INWIT (screenshot taken from the Autodesk Viewer web service).

\section{DEVELOPED METHODOLOGY}

\subsection{Concept and scope}

The project is carried on through a research contract between the Department of Architecture and Design of the Politecnico di Torino and the industrial partner INWIT - Infrastrutture Wireless Italiane S.p.A. INWIT operates an extensive network of antenna towers and related equipment. These assets have recently undergone a digitalization process, documenting the on-site condition through BIM and enhancing accessibility to models and data using a cloud platform (Figure 1).

The project has a twofold objective. On the one hand, we want to study methods for the automatic recognition of such assets to allow easy consultation of the information models, even for nonexpert operators. Using the physical object as a map for digital information (Spallone and Palma, 2021), one can quickly identify a component that needs intervention and obtain data and instructions. On the other and, the on-site interaction with the model allows a comparison with the current state of the site and helps to update existing models.

The sites managed by the company present a wide variety of typologies, but the project focused on a subset characterized by low-rise towers. Therefore, we expect that final users will operate on the ground and without the aid of special equipment. The targeted assets are antennas on poles - or small lattice towers and other ground equipment, including switchboards, power stations, and cabinets. These are generally standardized objects subject to frequent substitution, integration, and alteration.

\subsection{Components}

The research team developed semi-automatic systems for recognizing artifacts and updating and interrogating the existing BIM database, also addressed to non-expert users. Various technologies based on computer vision were examined including machine-learning object detection and mixed reality tools - to compose an effective system for identifying or recognizing the equipment present on the site. In particular, during the project, the choice fell on AR systems to obtain the site recognition and tracking, and explore its digital replica. Hence, we developed intuitive and rapid tools for browsing information and reporting discrepancies between the building site and the last model update.

The work consisted in developing a pipeline that allows communication between an AR app and the server containing the company's BIM database. The system was organized into three main components (Figure 2):

- an application programming interface (API) for communication between the app and the database;

- $\quad$ the graphic interface of the mobile app;

- the toolkits to enable AR, including AR functions on the app side and tools to produce and host target models.

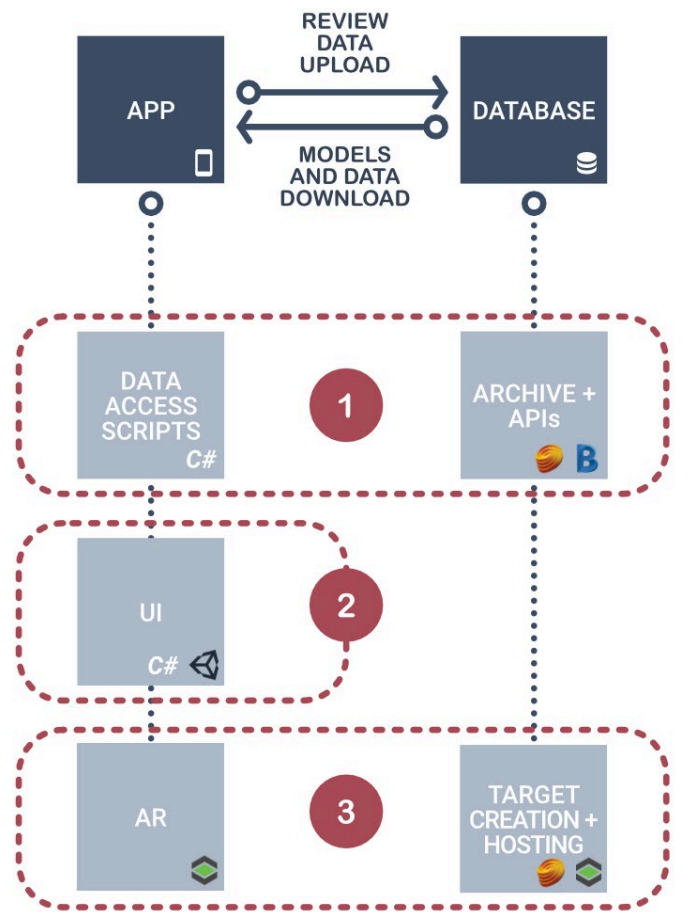

Figure 2. Scheme of the main components of the developed system: (1) server-client API communication; (2) user interface on the app side; (3) AR model archive and AR visualization tools. 


\subsection{Employed software}

The software used to develop AR functions, namely Vuforia by PTC Inc., allows the integration of sensor-based and vision-based tracking techniques and the recognition of natural features in three-dimensional objects. This makes it possible to test markerless systems and perform extended tracking, that is, to display the virtual overlay even far from a reference object (Amin and Govilkar, 2015). Furthermore, the system offers several options for established and reliable tracking techniques, such as image and barcode-like markers, which we used to develop backup solutions.

We created the structure and the mobile interface of the app using the Unity game engine, a popular development environment also for serious games and non-gaming applications that involve extensive use of 3D graphics (Kontogianni and Georgopoulos, 2015). Unity allowed us to develop a multi-platform app, create communication systems with a server, and download components at runtime through a network connection. The latter is an essential feature for a scalable app that requires both geometric models for visualization and site-specific datasets for activating $\mathrm{AR}$ functions.

Finally, the BIM model server used by INWIT is based on Autodesk cloud services, that is, the BIM 360 management software and Forge web APIs.

\section{PROTHOTYPING}

\subsection{App-database communication}

The first component enables communication with the BIM database. The app can access models and data on the company's servers and submit issues for reporting updates. The component required designing software modules both on the mobile application side and the server - to manage models.

The company's models are produced in Autodesk Revit and organized through the BIM 360 platform, a toolkit for project cloud management. Autodesk APIs Forge include solutions for interacting with the BIM 360 database, and therefore allow a third-party app to read documents and send data. The app (client) can send request messages to the server and get a response in text format (JSON). At the current stage of development, the APIs have been used to extract metadata from a Revit file on the server. We obtained the list of elements of a single test model, specifically uploaded to the server, and the attribute list of each one (Figure 3 ). The messages sent by the server are decoded by the app and sent to the interface components.
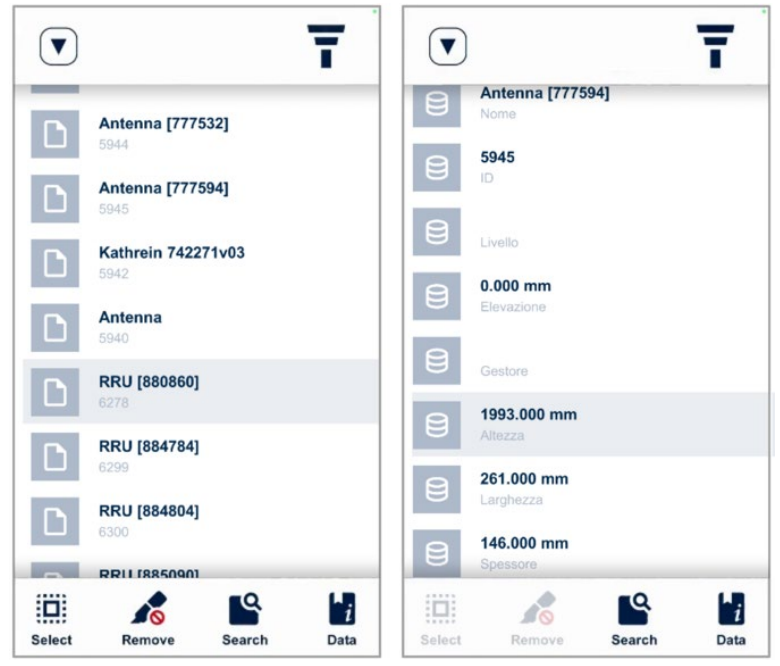

Figure 3. Screenshots of the data views of the developed app. The left view shows the list of available elements, while the right one shows the attributes of a single element.

The app needs to obtain, in addition to metadata, also the geometric information of Revit files, to be fully scalable and
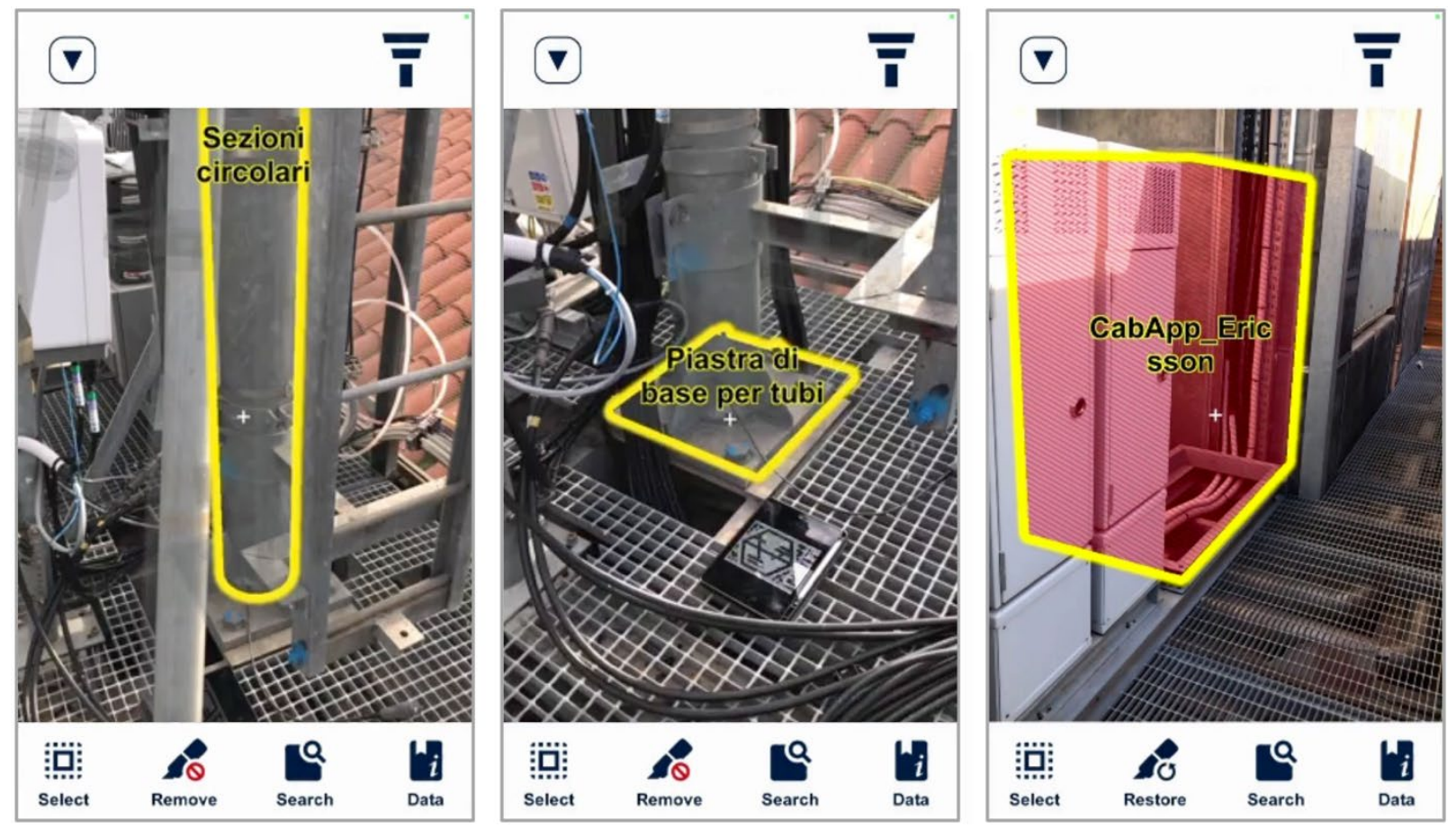

Figure 4. Screenshots of the developed mobile app, showing the selection of BIM elements through the camera view. In the central image, a tablet temporarily displays the tracked marker for test purposes; in the other images. The image on the left shows one of the developed markup tools, allowing the user to highlight a model element that is missing on-site. 
adaptable to an extensive and expanding BIM model archive. We tested the download of FBX files previously exported from BIM documents. The FBX models produced include each element's unique ID used in the Revit project (element's ID Number). In this way, once the app obtains the geometric model and the metadata, it can connect each element to its attributes. The display model corresponds to a subset of the elements of the Revit project since it excludes parts that are not useful for AR functions, such as small details and the masses representing the building that houses antennas.

However, the exposure of geometric data has yet to be optimized: it is necessary to create batch processes for exporting from large archives such as that of INWIT. Additionally, further tests will evaluate other APIs to directly extract geometry from the Revit file (for example, Forge's Model Derivative API allows this type of operation).

\subsection{Graphic interface}

The second component concerns the client device, that is, the mobile user interface solutions and the functions to make web services available.

The component includes the following sets of tools:

- tools for data visualization;

- $\quad$ tools for exploring the 3D model;

- tools for editing data to be sent to the server.

Data visualization is managed through cards featuring a list layout. The main views are the model elements view and the datasheet related to a single element.

We designed tools that make the 3D model easier to explore, combined with AR functions. In particular, a user can highlight some specific element, displaying its outline or a hatch pattern (Figure 4). The function can support reporting the deletion of an element (information to be forwarded to the server), finding objects selected through the list layout, or performing other manual selection and markup procedures. For example, an element could be highlighted upon notification of the database manager, provided that a specific parameter in the BIM project activates the selection in the frontend.

Model mesh rendering on top of the target object can be disabled through a visibility parameter. Displaying the camera view and the sole model-based outlines can produce the impression that the real object itself is being selected. The designed user interface (partly under construction) also includes on-screen arrows to search for objects outside the field of view, previously browsed through selection tools (Figure 5).
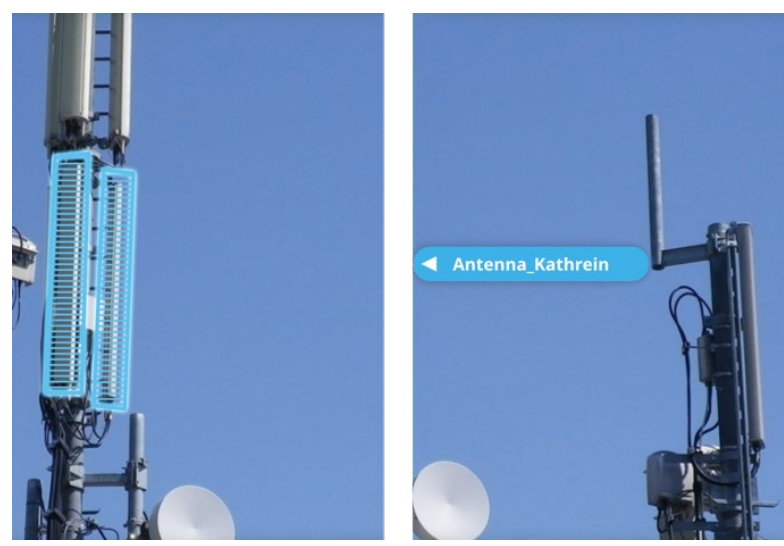

Figure 5. Graphic representation of one of the proposed user interface functions. When a selected object is off the field of view, an arrow on the edge of the screen can direct the user.

\subsection{AR: markerless experiments}

The AR component allows the visualization of the BIM model superimposed on the camera images. The preparation of this part of the prototype includes the development of the mobile app and a set of operations to set up AR targets, that is, the reference objects that the system uses to establish the relationship between real space and the virtual environment.

The project has provided for tests aimed at understanding whether, at present, commercial AR technologies, and the Vuforia SDK in particular, allow the recognition of threedimensional physical structures as reference points to enable the AR experience. Vuforia makes it possible to recognize and trace physical assets based on a 3D reproduction (Figure 6).

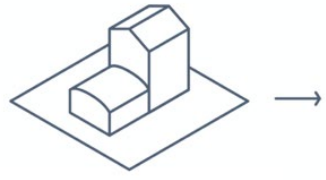

PHYSICAL OBJECT
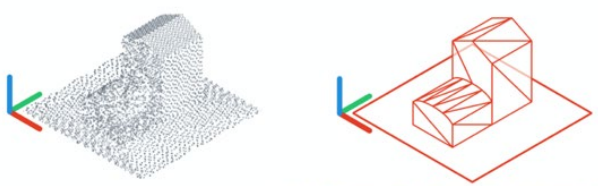

MODEL FOR VISUALIZATION

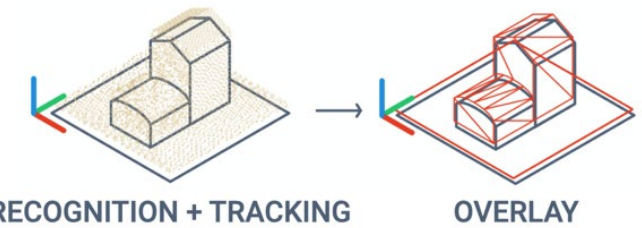

Figure 6. Scheme of the markerless AR tracking behavior.

Unity allows you to download specific software packages at runtime (UNITYPACKAGE files). These may include the information required by Vuforia to perform recognition functions, satisfying the need for application scalability.

The target can be a point cloud model or a mesh model. In both cases, Vuforia expects it to be converted into a compatible format through proprietary support tools. So far, the research has identified this step as a potentially critical issue for an efficient application, due to the manual work required by the model conversion processes. In fact, the tools made publicly available by Vuforia require several target optimization steps to be performed through a graphical interface (no command-line tools are provided).

Furthermore, the 3D targets showed some limitations. We produced mesh target models by filtering components of the Revit project to export models compatible with the conversion tools (supporting a maximum of 400000 polygons) such as FBX. Nevertheless, tests conducted on an INWIT site showed that the geometric inaccuracies of the current models do not deliver valid targets. In fact, many BIM documents were produced at the design stage and have not yet been updated to as-is conditions although INWIT has planned this improvement.

As for the creation of point cloud targets, the Vuforia software available at the time of testing (2021) was compatible with a limited set of acquisition tools (for example, Apple devices equipped with LiDAR or Matterport scanners) and mainly supported indoor acquisition procedures. These limits have not 
yet made it possible to carry out on-site tests, although future experiments will include validating the tracking of our open space models.

\subsection{AR: baseline solution}

To overcome 3D-target-related critical issues, the prototype was integrated with functions to recognize two-dimensional physical markers. We chose the barcode-like marker system provided by Vuforia, called VuMarks. These 2D markers have a highly customizable structure and encode data that the app can read during recognition (Figure 7). Our customized version records the site ID to let the model download start automatically. Among other advantages, the high contrast of the components, required by design constraints, guarantees effective recognition even with small target dimensions - the documentation suggests using targets larger than $25 \mathrm{~mm}$.

The drawbacks of this solution include the need to install one or more printed markers at each site. Since the target is point-like compared to model targets - more than one target may be needed to compensate for extended tracking errors if the user strays far from a target within a large site. In fact, in some contexts, the different pieces of equipment can have distances of tens of meters and also be arranged on several levels.

Markers require durable support, print, and anchoring system. Furthermore, the exact position of the target within the site must be measured and recorded in advance. We designed specific functions to survey target position - yet to be implemented again exploiting AR. The user will perform the survey after installing the physical target or before using the other functions of the app. In particular, one will be able to check through AR the correct alignment of the model and the site by viewing the model silhouette.
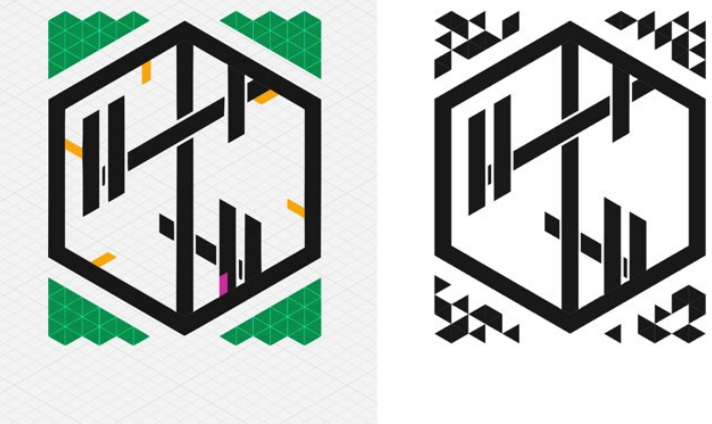

Figure 7. Example of marker developed for the INWIT sites, based on the Vuforia VuMark service. The left part of the image highlights components such as the variable pattern for code registration (green) and the relevant portions used to establish orientation (purple and yellow). Graphics inside the hexagonal border are not used for recognition purposes.

\section{RESULTS}

The project produced a prototype mobile app capable of superimposing in AR a 1:1 scale digital representation on the antenna towers and the connected equipment. The app, therefore, provides intuitive navigation of BIM documents. The prototype allows to select and interrogate the objects in the camera images, making the company's BIM models coincide with them. The user can query data and metadata and send reviews and reports to the BIM management team directly on-site through fast and easy-touse functions.
The main features of the designed app-database system are the following:

- it allows recognition and tracking of the tower site;

- is based on a cloud database, to support system scalability;

- allows interactions with the BIM database in reading and writing;

- includes a set of custom data visualization tools that facilitate interaction with the models.

Various solutions to implement the recognition and anchoring tools have been analyzed and tested, including markerless AR tracking techniques, highlighting some critical issues related to calibration, interoperability, and scalability, and allowing to define the possible development of the project.

\section{FUTURE DEVELOPMENTS}

The bidimensional marker system was designed as a backup solution for the markerless system, which will require further research and experiments to assess reliability. Although the AR functions with mesh targets require specific acquisition techniques, the pace of evolution of the tools allows us to predict an increase in the compatibility of conversion software with point cloud formats and acquisition tools. We can foresee that the future development phases of the project will also exploit point clouds associated with INWIT's assets - the company is conducting digital survey campaigns - and perform field tests to validate the effectiveness of targets of this type.

Other components of the project require further development. The sending of requests to the server for writing information on BIM models - issues for corrections and additions - has yet to be tested. We will also optimize the management of geometric files on the server, both for exporting display models and producing target models.

In the final stages of the prototype development, we will also evaluate the integration into the app of model visualization tools made available by BIM 360 (a viewer based on WebGL technology) to offer the user a virtual-only map of the site. Eventually, we will need to manage user authentication and monitor the status of requests. To implement these functions, Forge and BIM 360 tools are available, but custom interface elements will be required.

\section{CONCLUSIONS}

The current hype for AR enhances some technical possibilities of mobile computing and presents this technology as a naturally engaging and easy-to-use medium. However, several limitations seem to hinder the transformation into operational applications of the many methods, tests, and prototypes that are spreading in the AEC context (Sindani et al., 2021).

The presented work contributes to deepening aspects that still find little place in literature, such as the role of AR in the maintenance of both the physical object and an up-to-date model. The interaction with the industrial partner INWIT allowed to highlight the advantages of AR in managing the lifecycle of equipment and structures, in reducing errors during installation, and in reviewing design documents. In terms of technological advances in the AR field, the research stressed the advantages and limitations of solutions that use three-dimensional objects themselves as a target for setting up AR overlays.

In conclusion, the project, developed in a corporate context and aiming at a sound impact on the company's processes, contributes to the discussion on the benefits and costs of adopting AR technology, supporting the feasibility study of similar solutions in the AEC industry. 


\section{ACKNOLEDGEMENTS}

The project is carried out in the framework of a research contract between INWIT and Politecnico di Torino for the implementation of research projects. The project "Modellizzazione BIM automatica" (Automatic BIM modeling) started at the end of 2020 and was renewed for a second year of collaboration on the topics described. The team supervising the project at INWIT is composed by Luca Capozucca, Giulia Cicone, Gianpiero Lops, and Roberto Rinauro. The research team of the Department of Architecture and Design at Politecnico di Torino is composed by Roberta Spallone (supervisor) and Valerio Palma (research fellowship holder). The project is funded by INWIT. Figures 1, 3, 4 and 7 are courtesy of INWIT.

\section{REFERENCES}

Ahmed, S., 2019. A Review on Using Opportunities of Augmented Reality and Virtual Reality in Construction Project Management. Organization, Technology and Management in Construction: An International Journal, 11(1), pp. 1839-1852. https://doi.org/10.2478/otmcj-2018-0012

Akbari, A., 2018. Cyber-Physical Construction. IAAC Blog. http://www.iaacblog.com/programs/cyber-physicalconstruction/ (21 January 2022).

Amin, D., Govilkar, S., 2015. Comparative Study of Augmented Reality SDK's. International Journal on Computational Science \& Applications $\quad$ 5(1), 11-26. https://doi.org/10.5121/ijcsa.2015.5102.

Campi, M., di Luggo A., M. Falcone, M., 2021. Photogrammetric Processes and Augmented Reality Applications Using Mobile Devices. Int. Arch. Photogramm. Remote Sens. Spatial Inf. Sci, XLVI-M-1， 101-6. https://doi.org/10.5194/isprs-archivesXLVI-M-1-2021-101-2021.

Felix, J., 2019. Reality, but not as you know it. Building Connection, Summer 2019, 12-13. https://search.informit.org/doi/10.3316/informit.992006461166 235 (21 January 2022).

Garbett, J., Hartley, T., Heesom, D., 2021. A multi-user collaborative BIM-AR system to support design and construction. Automation in Construction, 122, 103487. https://doi.org/10.1016/j.autcon.2020.103487

Grieves, M., Vickers, J., 2017. Digital Twin: Mitigating Unpredictable, Undesirable Emergent Behavior in Complex Systems. In Kahlen, F. J., Flumerfelt, S., Alves, A. (Eds.). Transdisciplinary Perspectives on Complex Systems. Springer International Publishing, Cham, 85-113. https://doi.org/10.1007/978-3-319-38756-7_4.

Jahn, G., Newnham, C., van den Berg, N., Iraheta, M., Wells, J., 2020. Holographic Construction. In C. Gengnagel, O. Baverel, J. Burry, M. Ramsgaard Thomsen, S. Weinzierl (Eds.), Impact: Design with All Senses. Springer International Publishing, Cham, 314-324. https://doi.org/10.1007/978-3-030-29829-6 25

Khajavi, S.H., Motlagh, N.H., Jaribion A., Werner, L.C., Holmstrom, J., 2019. Digital Twin: Vision, Benefits, Boundaries, and Creation for Buildings. IEEE Access 7, 147406-19. https://doi.org/10.1109/ACCESS.2019.2946515.
Kopsida, M., Brilakis, I., 2016. Markerless BIM Registration for Mobile Augmented Reality Based Inspection. Proceedings of the International Conference on Smart Infrastructure and Construction, pp. 1631-1636. http://www.see.eng.osaka-u.ac.jp/ (21 January 2022).

Kontogianni, G., Georgopoulos, A., 2015. Exploiting Textured 3D Models for Developing Serious Games. Int. Arch. Photogramm. Remote Sens. Spatial Inf. Sci, XL-5/W7, 249-255. https://doi.org/10.5194/isprsarchives-XL-5-W7-249-2015.

Li, X., Yi, W., Chi, H.-L., Wang, X., Chan, A.P.C., 2018. A Critical Review of Virtual and Augmented Reality (VR/AR) Applications in Construction Safety. Automation in Construction 86, 150-162. https://doi.org/10.1016/j.autcon.2017.11.003.

Lin, G., Panigrahi, T., Womack, J., Ponda D. J., Kotipalli, P., Starner, T., 2021. Comparing Order Picking Guidance with Microsoft Hololens, Magic Leap, Google Glass XE and Paper. In Proceedings of the 22nd International Workshop on Mobile Computing Systems and Applications, 133-139. ACM. https://doi.org/10.1145/3446382.3448729.

Milgram, P., Kishino, F., 1994. A Taxonomy of Mixed Reality Visual Displays. IEICE TRANSACTIONS on Information and Systems, 77(12), 1321-1329.

Noghabaei, M., Heydarian, A., Balali, V., Han, K., 2020. Trend Analysis on Adoption of Virtual and Augmented Reality in the Architecture, Engineering, and Construction Industry. Data, 5(1), 26. https://doi.org/10.3390/data5010026

RealWear, Inc., 2021. Realwear and Autodesk break new ground with assisted reality construction project management solution. RealWear. https://www.realwear.com/pressreleases/permasteelisa-selects-realwear-autodesk-bim360/ (21 January 2022).

Sidani, A., Matoseiro Dinis, F., Duarte, J., Sanhudo, L., Calvetti, D., Santos Baptista, J., Poças Martins, J., Soeiro, A., 2021. Recent tools and techniques of BIM-Based Augmented Reality: A systematic review. Journal of Building Engineering, 42, 102500. https://doi.org/10.1016/j.jobe.2021.102500

Spallone, R., and Palma V, 2021. Artificial Intelligence and Augmented Reality: A Possible Continuum for the Enhancement of Architectural Heritage. DISEGNARECON 14, no. 26, 16.116.11. https://doi.org/10.20365/DISEGNARECON.26.2021.16.

Song, Y., Koeck, R., Luo, S., 2021. Review and Analysis of Augmented Reality (AR) Literature for Digital Fabrication in Architecture. Automation in Construction, 128, 103762. https://doi.org/10.1016/j.autcon.2021.103762.

Williams, G., Gheisari, M., Chen, P.-J., Irizarry, J., 2015. BIM2MAR: An Efficient BIM Translation to Mobile Augmented Reality Applications. Journal of Management in Engineering, 31(1). https://doi.org/10.1061/(ASCE)ME.1943-5479.0000315

Zaher, M., Greenwood, D., Marzouk, M. 2018. Mobile Augmented Reality Applications for Construction Projects. Construction Innovation 18(2), 152-166. https://doi.org/10.1108/CI-02-2017-0013. 